\title{
EXACT TRANSIENT SOLUTION OF A STATE-DEPENDENT BIRTH-DEATH PROCESS
}

\author{
P. R. PARTHASARATHY AND R. SUDHESH
}

Received 2 December 2004; Revised 12 May 2005; Accepted 13 May 2005

A power series expression in closed form for the transient probabilities of a state-dependent birth-death process is presented with suitable illustrations.

Copyright (c) 2006 P. R. Parthasarathy and R. Sudhesh. This is an open access article distributed under the Creative Commons Attribution License, which permits unrestricted use, distribution, and reproduction in any medium, provided the original work is properly cited.

\section{Introduction}

Continued fractions (CFs) play a fundamental role in many investigations due to its applications to diverse fields like number theory, special functions, approximations, moment problems, digital networks, statistics, and signal processing. Their importance has grown further with the advent of fast computing facilities.

The problem of converting a continued fraction into a power series is important in applications and has been studied by several authors for more than a century. The coefficients of a CF can be determined from the coefficients of a given power series through quotient of Hankel determinants (Vein and Dale [17]). Jones and Thron [7, page 227] describe a quotient-difference algorithm (qd algorithm) for computing the coefficients of continued fractions corresponding to a given power series. The J-fraction corresponding to a power series has been obtained from an addition formula by means of a decomposition (Goulden and Jackson [5, page 295]). Euler's connection describes an equivalence between a T-fraction and a power series (Gill [4]).

The converse relation to evaluate the power series coefficients from a known continued fraction expansion is pertinent and complicated. Rogers [15] obtained the first few coefficients and Ramanujan (Berndt [2, Entry 17]) has given a recursion. Wall [18, page 203] has presented an infinite Stieltjes matrix equation to obtain the coefficients of the power series expansion of a J-fraction. Zajta and Pandikow [19], Flajolet [3], and Goulden and Jackson [5] have employed a combinatorial approach.

CF applications to the study of birth and death processes (BDPs) were initiated by Murphy and O'Donohoe [9] and later this concept has been discussed by several authors 
(e.g., Guillemin and Pinchon [6]).This association has been exploited to find the timedependent solutions of certain BDPs in closed form for specific birth and death rates (Parthasarathy [11], Parthasarathy and Lenin [12]).

In this paper, we obtain the transient probabilities of state-dependent birth and death processes in terms of power series expression using CFs. In this study, the underlying forward Kolmogorov differential-difference equations are first transformed into a set of linear algebraic equations by employing Laplace transforms. This leads to a J-fraction which is expressed as a formal power series. Inverting we get the transient probabilities of state-dependent BDPs in closed form. Several examples are presented to illustrate this approach.

\section{CFs and BDPs}

Let $\{X(t), t \geq 0\}$ be a birth-death process with state-dependent birth and death rates $a_{2 n}$ and $a_{2 n+1}, n=0,1,2, \ldots$, respectively (instead of the usual $\lambda_{n}$ and $\mu_{n}$ ) defined on a probability space $(\Omega, \mathscr{F}, P)$. The reason for this notation will become apparent in the sequel. Then $P(X(t)=n \mid X(0)=k)=P_{k n}(t)$ satisfy the forward Kolmogorov equations:

$$
\begin{gathered}
P_{k 0}^{\prime}(t)=-a_{0} P_{k 0}(t)+a_{1} P_{k 1}(t), \\
P_{k n}^{\prime}(t)=a_{2 n-2} P_{k, n-1}(t)-\left(a_{2 n-1}+a_{2 n}\right) P_{k n}(t)+a_{2 n+1} P_{k, n+1}(t), \quad n=1,2,3, \ldots
\end{gathered}
$$

Taking Laplace transforms,

$$
f_{n}^{(k)}(s)=\int_{0}^{\infty} e^{-s t} P_{k n}(t) d t, \quad n=0,1,2, \ldots
$$

for $\operatorname{Re}(s)>0$ of the system of equations given by (2.1). Initially we assume that $k=0$, and in that case $f_{0}(s)$ simplifies to the expression

$$
\begin{gathered}
f_{0}(s)=\frac{1}{s+a_{0}-a_{1}\left(f_{1}(s) / f_{0}(s)\right)}, \\
\frac{f_{n}(s)}{f_{n-1}(s)}=\frac{a_{2 n-2}}{s+a_{2 n-1}+a_{2 n}-a_{2 n+1}\left(f_{n+1}(s) / f_{n}(s)\right)}, \quad n=1,2,3, \ldots, \\
= \\
\sqrt{s+a_{2 n-1}+a_{2 n}}-\frac{a_{2 n} a_{2 n+1}}{\mid s+a_{2 n+1}+a_{2 n+2}}-\frac{a_{2 n+2} a_{2 n+3}}{\mid s+a_{2 n+3}+a_{2 n+4}}-\ldots .
\end{gathered}
$$

Iterating this,

$$
f_{0}(s)=\frac{1}{\sqrt{s+a_{0}}}-\frac{a_{0} a_{1} J_{-}}{s^{s+a_{1}+a_{2}}}-\frac{a_{2} a_{3}}{s+a_{3}+a_{4}}-\cdots,
$$

where we use the notation

$$
\frac{a_{1} \mid}{\mid b_{1}}-\frac{a_{2} \mid}{\mid b_{2}}-\frac{a_{3} \mid}{\mid b_{3}}-\cdots=\frac{a_{1}}{b_{1}-\frac{a_{2}}{b_{2}-\frac{a_{3}}{b_{3}-}}} .
$$


This is a J-fraction (or Jacobi fraction) and it can be represented as an S-fraction:

$$
f_{0}(s)=\frac{1 / s \mid}{\mid 1}+\frac{a_{0} / s \mid}{\mid 1}+\frac{a_{1} / s \mid}{\mid 1}+\frac{a_{2} / s \mid}{\mid 1}+\frac{a_{3} / s \mid}{\mid 1}+\cdots .
$$

For notational convenience, we use $f_{n}(s)$ instead of $f_{n}^{(0)}(s)$ throughout this paper.

In the next section, we obtain power series expression for $P_{0 n}(t), n=0,1,2, \ldots$, using CFs.

\section{Transient probabilities}

In this section, we express the J-fraction (2.4) as a formal power series leading to the state probabilities of general state-dependent BDPs. First we prove the following result.

Theorem 3.1. If

$$
\frac{f_{n}(s)}{f_{n-1}(s)}=a_{2 n-2} \sum_{m=0}^{\infty}(-1)^{m} B(m, n) \frac{1}{s^{m+1}},
$$

then $B(0, n)=1$ and for $m=1,2,3, \ldots$,

$$
B(m, n)=\sum_{i_{1}=2 n-1}^{2 n} a_{i_{1}} \sum_{i_{2}=2 n-1}^{i_{1}+1} a_{i_{2}} \sum_{i_{3}=2 n-1}^{i_{2}+1} a_{i_{3}} \cdots \sum_{i_{m}=2 n-1}^{i_{m-1}+1} a_{i_{m}} \quad \forall n \in \mathbb{N} .
$$

Proof. Let us assume that

$$
\frac{f_{n+1}(s)}{f_{n}(s)}=a_{2 n} \sum_{m=0}^{\infty}(-1)^{m} B(m, n+1) \frac{1}{s^{m+1}} .
$$

Also,

$$
\begin{aligned}
\frac{f_{n}(s)}{f_{n-1}(s)} & =\frac{a_{2 n-2}}{s+a_{2 n-1}+a_{2 n}-a_{2 n+1}\left(f_{n+1}(s) / f_{n}(s)\right)} \\
& =\frac{a_{2 n-2}}{s+a_{2 n-1}+a_{2 n}-a_{2 n} a_{2 n+1} \sum_{m=0}^{\infty}(-1)^{m} B(m, n+1)\left(1 / s^{m+1}\right)} .
\end{aligned}
$$

From (3.1) and (3.4),

$$
\left[\sum_{m=0}^{\infty}(-1)^{m} B(m, n) \frac{1}{s^{m+1}}\right]\left[1+\frac{a_{2 n-1}+a_{2 n}}{s}-a_{2 n} a_{2 n+1} \sum_{m=0}^{\infty}(-1)^{m} B(m, n+1) \frac{1}{s^{m+2}}\right]=\frac{1}{s} .
$$

In the above Cauchy product, only the coefficient of $1 / s$ survives and the remaining coefficients will be zero. For every $n$ and $m=1,2,3, \ldots$, we obtain the following relation:

$$
B(m, n)=\left(a_{2 n-1}+a_{2 n}\right) B(m-1, n)+a_{2 n} a_{2 n+1}\left[\sum_{k=0}^{m-2} B(k, n+1) B(m-k-2, n)\right] .
$$

From this, we prove the result (3.2) by induction on $m$ for every $n$. 
4 Transient solution of state-dependent BDPs

For $m=1$,

$$
B(1, n)=a_{2 n-1}+a_{2 n}=\sum_{i_{1}=2 n-1}^{2 n} a_{i_{1}} \text { for every } n .
$$

Assume that for every $n$, this result is valid for each nonnegative integer up to $m-1$. That is,

$$
B(m-1, n)=\sum_{i_{1}=2 n-1}^{2 n} a_{i_{1}} \sum_{i_{2}=2 n-1}^{i_{1}+1} a_{i_{2}} \sum_{i_{3}=2 n-1}^{i_{2}+1} a_{i_{3}} \cdots \sum_{i_{m-1}=2 n-1}^{i_{m-2}+1} a_{i_{m-1}} \quad \forall n \in \mathbb{N} .
$$

Since

$$
\begin{gathered}
B(1, n+1) B(m-3, n)+B(2, n+1) B(m-4, n)+\cdots+B(m-2, n+1) \\
=\sum_{i_{1}=2 n+1}^{2 n+2} a_{i_{1}} \sum_{i_{2}=2 n-1}^{i_{1}+1} a_{i_{2}} \sum_{i_{3}=2 n-1}^{i_{2}+1} a_{i_{3}} \cdots \sum_{i_{m-2}=2 n-1}^{i_{m-3}+1} a_{i_{m-2}},
\end{gathered}
$$

then

$$
\begin{aligned}
B(m- & 2, n)+B(1, n+1) B(m-3, n)+B(2, n+1) B(m-4, n)+\cdots+B(m-2, n+1) \\
& =\sum_{i_{1}=2 n-1}^{2 n} a_{i_{1}} \sum_{i_{2}=2 n-1}^{i_{1}+1} a_{i_{2}} \ldots \sum_{i_{m-2}=2 n-1}^{i_{m-3}+1} a_{i_{m-2}}+\sum_{i_{1}=2 n+1}^{2 n+2} a_{i_{1}} \sum_{i_{2}=2 n-1}^{i_{1}+1} a_{i_{2}} \cdots \sum_{i_{m-2}=2 n-1}^{i_{m-3}+1} a_{i_{m-2}} \\
& =\sum_{i_{1}=2 n-1}^{2 n+2} a_{i_{1}} \sum_{i_{2}=2 n-1}^{i_{1}+1} a_{i_{2}} \sum_{i_{3}=2 n-1}^{i_{2}+1} a_{i_{3}} \ldots \sum_{i_{m-2}=2 n-1}^{i_{m-3}+1} a_{i_{m-2}} .
\end{aligned}
$$

Therefore,

$$
\begin{aligned}
B(m, n)= & a_{2 n-1} B(m-1, n)+a_{2 n} B(m-1, n)+a_{2 n} a_{2 n+1}\left[\sum_{k=0}^{m-2} B(k, n+1) B(m-k-2, n)\right] \\
= & a_{2 n-1} \sum_{i_{1}=2 n-1}^{2 n} a_{i_{1}} \sum_{i_{2}=2 n-1}^{i_{1}+1} a_{i_{2}} \sum_{i_{3}=2 n-1}^{i_{2}+1} a_{i_{3}} \ldots \sum_{i_{m-1}=2 n-1}^{i_{m-2}+1} a_{i_{m-1}} \\
& +a_{2 n} \sum_{i_{1}=2 n-1}^{2 n} a_{i_{1}} \sum_{i_{2}=2 n-1}^{i_{1}+1} a_{i_{2}} \sum_{i_{3}=2 n-1}^{i_{2}+1} a_{i_{3}} \ldots \sum_{i_{m-1}=2 n-1}^{i_{m-2}+1} a_{i_{m-1}} \\
& +a_{2 n} a_{2 n+1} \sum_{i_{1}=2 n-1}^{2 n+2} a_{i_{1}} \sum_{i_{2}=2 n-1}^{i_{1}+1} a_{i_{2}} \sum_{i_{3}=2 n-1}^{i_{2}+1} a_{i_{3}} \ldots \sum_{i_{m-2}=2 n-1}^{i_{m-3}+1} a_{i_{m-2}}
\end{aligned}
$$




$$
\begin{aligned}
& =a_{2 n-1} \sum_{i_{1}=2 n-1}^{2 n} a_{i_{1}} \sum_{i_{2}=2 n-1}^{i_{1}+1} a_{i_{2}} \sum_{i_{3}=2 n-1}^{i_{2}+1} a_{i_{3}} \ldots \sum_{i_{m-1}=2 n-1}^{i_{m-2}+1} a_{i_{m-1}} \\
& +a_{2 n} \sum_{i_{1}=2 n-1}^{2 n+1} a_{i_{1}} \sum_{i_{2}=2 n-1}^{i_{1}+1} a_{i_{2}} \sum_{i_{3}=2 n-1}^{i_{2}+1} a_{i_{3}} \ldots \sum_{i_{m-1}=2 n-1}^{i_{m-2}+1} a_{i_{m-1}} \\
& =\sum_{i_{1}=2 n-1}^{2 n} a_{i_{1}} \sum_{i_{2}=2 n-1}^{i_{1}+1} a_{i_{2}} \sum_{i_{3}=2 n-1}^{i_{2}+1} a_{i_{3}} \cdots \sum_{i_{m}=2 n-1}^{i_{m-1}+1} a_{i_{m}} .
\end{aligned}
$$

Thus the result is true for $m$.

Expression for $P_{00}(t)$. The next theorem gives an expansion of J-fraction as a power series with explicit coefficients.

THeOREM 3.2. If

$$
P_{00}(t)=\sum_{m=0}^{\infty}(-1)^{m} A(m, 0) \frac{t^{m}}{m !}
$$

then $A(0,0)=1$ and for $m=1,2,3, \ldots$,

$$
A(m, 0)=a_{0} \sum_{i_{1}=0}^{1} a_{i_{1}} \sum_{i_{2}=0}^{i_{1}+1} a_{i_{2}} \sum_{i_{3}=0}^{i_{2}+1} a_{i_{3}} \cdots \sum_{i_{m-1}=0}^{i_{m-2}+1} a_{i_{m-1}} .
$$

Proof. Let us assume that

$$
f_{0}(s)=\sum_{m=0}^{\infty}(-1)^{m} \frac{A(m, 0)}{s^{m+1}}
$$

From (3.1),

$$
f_{0}(s)=\frac{1}{s+a_{0}-a_{1}\left(f_{1}(s) / f_{0}(s)\right)}=\frac{1}{s+a_{0}-a_{0} a_{1} \sum_{m=0}^{\infty}(-1)^{m}\left(B(m, 1) / s^{m+1}\right)} .
$$

From (3.14) and (3.15),

$$
\left[\sum_{m=0}^{\infty}(-1)^{m} \frac{A(m, 0)}{s^{m+1}}\right]\left[1+\frac{a_{0}}{s}-a_{0} a_{1} \sum_{m=0}^{\infty}(-1)^{m} \frac{B(m, 1)}{s^{m+2}}\right]=\frac{1}{s} .
$$

Hence for $m=1,2,3, \ldots$,

$$
A(m, 0)=a_{0} A(m-1,0)+a_{0} a_{1}\left[\sum_{k=0}^{m-2} B(k, 1) A(m-k-2,0)\right] .
$$

Now, we prove the result (3.13) by induction on $m$. Using (3.17), it is easily seen that 
6 Transient solution of state-dependent BDPs

for $m=1,2$,

$$
\begin{gathered}
A(1,0)=a_{0} ; \\
A(2,0)=a_{0} A(1,0)+a_{0} a_{1} A(0,0)=a_{0}\left(a_{0}+a_{1}\right)=a_{0} \sum_{i_{1}=0}^{1} a_{i_{1}} .
\end{gathered}
$$

Assume that this result is valid for each nonnegative integer up to $m$ :

$$
A(m, 0)=a_{0} \sum_{i_{1}=0}^{1} a_{i_{1}} \sum_{i_{2}=0}^{i_{1}+1} a_{i_{2}} \sum_{i_{3}=0}^{i_{2}+1} a_{i_{3}} \cdots \sum_{i_{m-1}=0}^{i_{m-2}+1} a_{i_{m-1}} .
$$

Using (3.2) in (3.17),

$$
\begin{aligned}
A(m+1,0)= & a_{0} A(m, 0)+a_{0} a_{1}[A(m-1,0)+A(m-2,0) B(1,1)+A(m-3,0) B(2,1) \\
& +\cdots+A(1,0) B(m-2,1)+B(m-1,1)] \\
= & a_{0} a_{0} \sum_{i_{1}=0}^{1} a_{i_{1}} \sum_{i_{2}=0}^{i_{1}+1} a_{i_{2}} \sum_{i_{3}=0}^{i_{2}+1} a_{i_{3}} \cdots \sum_{i_{m-1}=0}^{i_{m-2}+1} a_{i_{m-1}} \\
& +a_{0} a_{1}\left[a_{0} \sum_{i_{1}=0}^{1} a_{i_{1}} \sum_{i_{2}=0}^{i_{1}+1} a_{i_{2}} \sum_{i_{3}=0}^{i_{2}+1} a_{i_{3}} \cdots \sum_{i_{m-2}=0}^{i_{m-3}+1} a_{i_{m-2}}\right. \\
& \left.+\sum_{i_{1}=1}^{2} a_{i_{1}} \sum_{i_{2}=0}^{i_{1}+1} a_{i_{2}} \sum_{i_{3}=0}^{i_{2}+1} a_{i_{3}} \cdots \sum_{i_{m-1}=0}^{i_{m-2}+1} a_{i_{m-1}}\right] \\
= & a_{0} a_{0} \sum_{i_{1}=0}^{1} a_{i_{1}} \sum_{i_{2}=0}^{i_{1}+1} a_{i_{2}} \sum_{i_{3}=0}^{i_{2}+1} a_{i_{3}} \ldots \sum_{i_{m-1}=0}^{i_{m-2}+1} a_{i_{m-1}} \\
& +a_{0} a_{1} \sum_{i_{1}=0}^{2} a_{i_{1}} \sum_{i_{2}=0}^{i_{1}+1} a_{i_{2}} \sum_{i_{3}=0}^{i_{2}+1} a_{i_{3}} \ldots \sum_{i_{m-1}=0}^{i_{m-2}+1} a_{i_{m-1}} .
\end{aligned}
$$

Hence the result is true for $m+1$.

From (3.14), we obtain (3.12).

Expression for $P_{0 n}(t)$. The next theorem gives expressions for $P_{0 n}(t), n=1,2,3, \ldots$.

Theorem 3.3. For $n=1,2,3, \ldots$,

$$
P_{0 n}(t)=L_{n-1} \sum_{m=0}^{\infty}(-1)^{m} A(m, 2 n) \frac{t^{m+n}}{(m+n) !},
$$

where

$$
A(m, n)=\sum_{i_{1}=0}^{n} a_{i_{1}} \sum_{i_{2}=0}^{i_{1}+1} a_{i_{2}} \sum_{i_{3}=0}^{i_{2}+1} a_{i_{3}} \cdots \sum_{i_{m}=0}^{i_{m-1}+1} a_{i_{m}} \quad \forall n \in \mathbb{N}
$$

and $L_{n-1}=a_{0} a_{2} a_{4} \cdots a_{2 n-2}, L_{-1}=1$ with $A(0, n)=1$. 
Proof. Theorems 3.1 and 3.2 yield

$$
\begin{aligned}
f_{1}(s)=\frac{f_{1}(s)}{f_{0}(s)} f_{0}(s) & =a_{0} \sum_{m=0}^{\infty}(-1)^{m} \frac{B(m, 1)}{s^{m+1}} \sum_{m=0}^{\infty}(-1)^{m} \frac{A(m, 0)}{s^{m+1}} \\
& =a_{0} \sum_{m=0}^{\infty} \frac{(-1)^{m}}{s^{m+2}} \sum_{k=0}^{m} A(m-k, 0) B(k, 1) \\
& =a_{0} \sum_{m=0}^{\infty}(-1)^{m} \frac{A(m, 2)}{s^{m+2}}
\end{aligned}
$$

where $A(m, 2)=\sum_{k=0}^{m} A(m-k, 0) B(k, 1)$.

From (3.2) and (3.13),

$$
A(m, 2)=\sum_{i_{1}=0}^{2} a_{i_{1}} \sum_{i_{2}=0}^{i_{1}+1} a_{i_{2}} \sum_{i_{3}=0}^{i_{2}+1} a_{i_{3}} \cdots \sum_{i_{m}=0}^{i_{m-1}+1} a_{i_{m}} .
$$

For $k=0$ and $n=1,2,3, \ldots$, the Laplace transform of (2.1) is given by

$$
\left(s+a_{2 n-1}+a_{2 n}\right) f_{n}(s)=a_{2 n-2} f_{n-1}(s)+a_{2 n+1} f_{n+1}(s) .
$$

Assume that, the power series representation of $f_{n}(s)$ is of the form

$$
f_{n}(s)=L_{n-1} \sum_{m=0}^{\infty}(-1)^{m} \frac{A(m, 2 n)}{s^{m+n+1}} .
$$

Substitute (3.26) in (3.25) and comparing the coefficients on each side, for $m \geq 2$ and for every $n$,

$$
A(m, 2 n)=A(m, 2 n-2)+\left(a_{2 n-1}+a_{2 n}\right) A(m-1,2 n)+a_{2 n} a_{2 n+1} A(m-2,2 n+2),
$$

and if $m=1$,

$$
A(1,2 n)=A(1,2 n-2)+\left(a_{2 n-1}+a_{2 n}\right) .
$$

From this,

$$
A(1,2 n)=\sum_{i_{1}=0}^{2 n} a_{i_{1}} \quad \text { for every } n
$$

By induction hypothesis, we assume that for each nonnegative integer $n$, this result is valid up to $m-1$, that is,

$$
A(m-1,2 n)=\sum_{i_{1}=0}^{2 n} a_{i_{1}} \sum_{i_{2}=0}^{i_{1}+1} a_{i_{2}} \sum_{i_{3}=0}^{i_{2}+1} a_{i_{3}} \cdots \sum_{i_{m-1}=0}^{i_{m-2}+1} a_{i_{m-1}} .
$$


Thus,

$$
\begin{aligned}
A(m, 2 n)= & A(m, 2 n-2)+\left(a_{2 n-1}+a_{2 n}\right) A(m-1,2 n)+a_{2 n} a_{2 n+1} A(m-2,2 n+2) \\
= & A(m, 2 n-2)+a_{2 n-1} \sum_{i_{1}=0}^{2 n} a_{i_{1}} \sum_{i_{2}=0}^{i_{1}+1} a_{i_{2}} \cdots \sum_{i_{m-1}=0}^{i_{m-2}+1} a_{i_{m-1}} \\
& +a_{2 n} \sum_{i_{1}=0}^{2 n} a_{i_{1}} \sum_{i_{2}=0}^{i_{1}+1} a_{i_{2}} \ldots \sum_{i_{m-1}=0}^{i_{m-2}+1} a_{i_{m-1}}+a_{2 n} a_{2 n+1} \sum_{i_{1}=0}^{2 n+2} a_{i_{1}} \sum_{i_{2}=0}^{i_{1}+1} a_{i_{2}} \ldots \sum_{i_{m-2}=0}^{i_{m-3}+1} a_{i_{m-2}} \\
= & A(m, 2 n-2)+a_{2 n-1} \sum_{i_{1}=0}^{2 n} a_{i_{1}} \sum_{i_{2}=0}^{i_{1}+1} a_{i_{2}} \cdots \sum_{i_{m-1}=0}^{i_{m-2}+1} a_{i_{m-1}} \\
& +a_{2 n} \sum_{i_{1}=0}^{2 n+1} a_{i_{1}} \sum_{i_{2}=0}^{i_{1}+1} a_{i_{2}} \cdots \sum_{i_{m-1}=0}^{i_{m-2}+1} a_{i_{m-1}} .
\end{aligned}
$$

Using $A(m, 0)=a_{0} \sum_{i_{1}=0}^{1} a_{i_{1}} \sum_{i_{2}=0}^{i_{1}+1} a_{i_{2}} \sum_{i_{3}=0}^{i_{2}+1} a_{i_{3}} \cdots \sum_{i_{m-1}=0}^{i_{m-2}+1} a_{i_{m-1}}$, the result is true for $m$.

We obtain (3.21) from (3.26).

Observe that the power series expression of $P_{0 n}(t)$ starts with $t^{n}$ and the coefficients $A(m, 2 n)$ and $B(m, n)$ differ only in the lower limits of the summations.

Expression for $P_{k n}(t)$. In the above analysis, we assumed that $P(X(0)=0)=1$. If $P(X(0)=$ $k)=1$,

$$
f_{n}^{(k)}(s)= \begin{cases}\frac{M_{k}}{L_{k-1} M_{n}} B_{n}(s) f_{k}(s), & n=0,1,2, \ldots, k, \\ \frac{1}{L_{k-1}} B_{k}(s) f_{n}(s), & n=k, k+1, k+2, \ldots \text { (see Jones and Thron [7]), }\end{cases}
$$

where $M_{n}=a_{1} a_{3} a_{5} \cdots a_{2 n-1}$ with $M_{0}=1$ and $B_{n}(s)$ is an orthogonal polynomial satisfying the three-term recurrence relation

$$
B_{n}(s)=\left(s+a_{2 n-3}+a_{2 n-2}\right) B_{n-1}(s)-a_{2 n-4} a_{2 n-3} B_{n-2}(s), \quad n=2,3,4, \ldots, k,
$$

with $B_{0}(s)=1$ and $B_{1}(s)=s+a_{0}$.

We can write

$$
B_{n}(s)=\sum_{m=0}^{n} \phi_{m}(2 n+1) s^{n-m}
$$

where $\phi_{0}(n)=1, \phi_{m}(n)=0$ if $m \geq[(n+1) / 2]$, for $n=1,2,3, \ldots$ and $m \geq 1$,

$$
\phi_{m}(n+1)=\sum_{i_{1}=0}^{n-2 m} a_{i_{1}} \sum_{i_{2}=i_{1}+2}^{n-2 m+2} a_{i_{2}} \sum_{i_{3}=i_{2}+2}^{n-2 m+4} a_{i_{3}} \cdots \sum_{i_{m}=i_{m-1}+2}^{n-2} a_{i_{m}}
$$

(see Parthasarathy and Sudhesh [14]). 
From (3.26) and (3.34),

$$
f_{n}^{(k)}(s)= \begin{cases}\frac{M_{k}}{M_{n}} \sum_{m=0}^{\infty} \frac{(-1)^{m}}{s^{m+k-n+1}} \sum_{r=0}^{\min (m, n)}(-1)^{r} \phi_{r}(2 n+1) A(m-r, 2 k), & n=0,1,2, \ldots, k, \\ \frac{L_{n-1}}{L_{k-1}} \sum_{m=0}^{\infty} \frac{(-1)^{m}}{s^{m+n-k+1}} \sum_{r=0}^{\min (m, k)}(-1)^{r} \phi_{r}(2 k+1) A(m-r, 2 n), & n=k, k+1, k+2, \ldots\end{cases}
$$

Inverting we get the following theorem.

THEOREM 3.4. If $X(0)=k$, then the power series representation for the state probabilities $P_{k n}(t), n=0,1,2, \ldots$, corresponding to a state-dependent BDP with birth and death rates, respectively, $a_{2 n}$ and $a_{2 n+1}(0 \leq n<\infty)$ are given by

$$
P_{k n}(t)=\left\{\begin{array}{l}
\frac{M_{k}}{M_{n}} \sum_{m=0}^{\infty}(-1)^{m} \frac{t^{m+k-n}}{(m+k-n) !} \\
\quad \times \sum_{r=0}^{\min (m, n)}(-1)^{r} \phi_{r}(2 n+1) A(m-r, 2 k), \quad n=0,1,2, \ldots, k, \\
\frac{L_{n-1}}{L_{k-1}} \sum_{m=0}^{\infty}(-1)^{m} \frac{t^{m+n-k}}{(m+n-k) !} \\
\quad \times \sum_{r=0}^{\min (m, k)}(-1)^{r} \phi_{r}(2 k+1) A(m-r, 2 n), \quad n=k, k+1, \ldots
\end{array}\right.
$$

\section{Examples}

From (3.22),

$$
\begin{aligned}
A(m, n) & =\sum_{i_{1}=0}^{n} a_{i_{1}} \sum_{i_{2}=0}^{i_{1}+1} a_{i_{2}} \sum_{i_{3}=0}^{i_{2}+1} a_{i_{3}} \cdots \sum_{i_{m}=0}^{i_{m-1}+1} a_{i_{m}} \\
& =\sum_{i_{1}=0}^{n-1} a_{i_{1}} \sum_{i_{2}=0}^{i_{1}+1} a_{i_{2}} \cdots \sum_{i_{m}=0}^{i_{m-1}+1} a_{i_{m}}+a_{n} \sum_{i_{2}=0}^{n+1} a_{i_{2}} \sum_{i_{3}=0}^{i_{2}+1} a_{i_{3}} \cdots \sum_{i_{m}=0}^{i_{m-1}+1} a_{i_{m}} \\
& =A(m, n-1)+a_{n} A(m-1, n+1) .
\end{aligned}
$$

Thus,

$$
A(m, n)=a_{0} A(m-1,1)+a_{1} A(m-1,2)+\cdots+a_{n} A(m-1, n+1) .
$$

This recurrence relation is useful to obtain elegant power series coefficients as illustrated below.

Example 4.1 (BDPs with equal rates). Let us assume that the birth and death rates are equal. That is, $a_{2 n}=a_{2 n+1}=\lambda>0, n=0,1,2, \ldots$ (i.e., $\lambda=\mu$ ). 
Then,

$$
A(m, n)=\frac{n+1}{n+m+1}\left(\begin{array}{c}
n+2 m \\
m
\end{array}\right) \lambda^{m} \text { for every } n
$$

Therefore,

$$
A(m, 2 n)=\frac{2 n+1}{2 n+m+1}\left(\begin{array}{c}
2 n+2 m \\
m
\end{array}\right) \lambda^{m}=\mathscr{C}_{m+n, n} \lambda^{m}
$$

where $\mathscr{C}_{m, n}=((2 n+1) /(n+m+1))\left(\begin{array}{c}2 m \\ m+n\end{array}\right), 0 \leq n \leq m$ is a generalized Catalan number.

If $a_{i}=\lambda, i=0,1,2, \ldots$, then

$$
f_{0}(s)=\frac{1 / s}{\Gamma 1}+\frac{\lambda / s}{\lceil 1}+\frac{\lambda / s}{\lceil 1}+\frac{\lambda / s}{\lceil 1}+\cdots=\sum_{m=0}^{\infty}(-1)^{m} \mathscr{C}_{m, 0} \frac{\lambda^{m}}{s^{m+1}},
$$

where $\mathscr{b}_{m, 0}=1 /(m+1)\left(\begin{array}{c}2 m \\ m\end{array}\right)$ is a Catalan number.

Thus, for $n=0,1,2, \ldots$,

$$
P_{0 n}(t)=\sum_{m=0}^{\infty}(-1)^{m} \mathscr{C}_{m+n, n} \frac{(\lambda t)^{m+n}}{(m+n) !} .
$$

Example 4.2 (busy period distribution of $\mathrm{M} / \mathrm{M} / 1$ queue). Customers arrive at an $\mathrm{M} / \mathrm{M} / 1$ queueing system at a Poisson process with parameter $\lambda$ and service times are exponentially distributed with parameter $\mu$. A busy period begins with the arrival of a customer to an idle channel and ends when the channel becomes idle once more. The cumulative distribution function of the busy period is determined by considering the original differential-difference equations for a birth-death process given in (2.1) with an absorbing barrier imposed at zero system size (i.e., $a_{0}=0$ ) and $a_{2 n}=\lambda, a_{2 n-1}=\mu, n=1,2,3, \ldots$.

Then $P_{0}^{\prime}(t)$ gives the density function of the length of the busy period. The differentialdifference equations are

$$
\begin{gathered}
P_{0}^{\prime}(t)=\mu P_{1}(t) \\
P_{1}^{\prime}(t)=-(\lambda+\mu) P_{1}(t)+\mu P_{2}(t), \\
P_{n}^{\prime}(t)=\lambda P_{n-1}(t)-(\lambda+\mu) P_{n}(t)+\mu P_{n+1}(t), \quad n=2,3,4, \ldots,
\end{gathered}
$$

with $P_{1}(0)=1$.

The Laplace transform of the busy period density function equals

$$
\left.\frac{\left.\mu\right|_{-}}{\mid s+\lambda+\mu}\right|_{-} \frac{\lambda \mu}{\mid s+\lambda+\mu}-\cdots=\frac{p-\sqrt{p^{2}-4 \lambda \mu}}{2 \lambda},
$$

where $p=s+\lambda+\mu$.

From this, the density function of the busy period equals $\sqrt{\mu / \lambda} e^{-(\lambda+\mu) t}\left(I_{1}[2 t \sqrt{\lambda \mu}] / t\right)$, $t>0$, where $I_{n}(\cdot)$ is the modified Bessel function of the first kind of order $n$. 
Example 4.3 (M/M/1 queue). The arrival (birth) and departure (death) rates are given by

$$
a_{2 n}=\lambda, \quad a_{2 n+1}=\mu, \quad n=0,1,2, \ldots
$$

From (2.4), for an $M / M / 1$ queue, $n=1,2,3, \ldots$,

$$
\begin{aligned}
\frac{f_{n}(s)}{f_{n-1}(s)} & =\frac{\lambda}{s+\lambda+\mu}-\frac{\lambda \mu}{s+\lambda+\mu}-\frac{\lambda \mu}{s+\lambda+\mu}-\cdots \\
& =\beta\left[\frac{p-\sqrt{p^{2}-\alpha^{2}}}{\alpha}\right],
\end{aligned}
$$

where $p=s+\lambda+\mu, \alpha=2 \sqrt{\lambda \mu}, \beta=\sqrt{\lambda / \mu}$.

Therefore,

$$
\begin{aligned}
& f_{n}(s)=\beta^{n}\left[\frac{p-\sqrt{p^{2}-\alpha^{2}}}{\alpha}\right]^{n} f_{0}(s), \\
& f_{0}(s)=\frac{1}{s}-\frac{\lambda}{s} \frac{2}{\alpha}\left[\frac{p-\sqrt{p^{2}-\alpha^{2}}}{\alpha}\right] .
\end{aligned}
$$

Thus, for $n=1,2,3, \ldots$,

$$
\begin{gathered}
P_{0 n}(t)=\lambda \beta^{n-1} \int_{0}^{t} \exp [-(\lambda+\mu) y]\left[I_{n-1}(\alpha y)-I_{n+1}(\alpha y)\right] P_{00}(t-y) d y, \\
P_{00}(t)=1-\lambda \int_{0}^{t} e^{-(\lambda+\mu) y}\left[I_{0}(\alpha y)-I_{2}(\alpha y)\right] d y
\end{gathered}
$$

(see Parthasarathy [10]).

Example 4.4 (chain sequence). We consider a birth-death process with birth and death rates $a_{2 n}$ and $a_{2 n+1}, n=0,1,2, \ldots$, satisfying the conditions

$$
a_{0}=1, \quad a_{2 n-1}+a_{2 n}=1, \quad a_{2 n-2} a_{2 n-1}=\gamma>0, \quad n=1,2,3, \ldots
$$

Then the birth and death rates are given by

$$
a_{2 n}=\frac{\tau U_{n+1}(1 / \tau)}{2 U_{n}(1 / \tau)}, \quad n=0,1,2, \ldots, \quad a_{2 n-1}=\frac{\tau U_{n-1}(1 / \tau)}{2 U_{n}(1 / \tau)}, \quad n=1,2,3, \ldots,
$$

where $\tau=2 \sqrt{\gamma}$.

The CF representation of $f_{0}(s)$ is given by

$$
f_{0}(s)=\frac{1}{\mid s+1}-\frac{\gamma}{\mid s+1}-\frac{\gamma}{\mid s+1}-\cdots=\frac{2}{\tau} \frac{p-\sqrt{p^{2}-\tau^{2}}}{\tau},
$$

where $p=s+1$. 
12 Transient solution of state-dependent BDPs

Also for $n=1,2,3, \ldots$,

$$
\frac{f_{n}(s)}{f_{n-1}(s)}=\frac{2 a_{2 n-2}}{\tau} \frac{p-\sqrt{p^{2}-\tau^{2}}}{\tau}
$$

and therefore,

$$
f_{n}(s)=\frac{2}{\tau} U_{n}\left(\frac{1}{\tau}\right)\left(\frac{p-\sqrt{p^{2}-\tau^{2}}}{\tau}\right)^{n+1} .
$$

From this for $n=0,1,2, \ldots$,

$$
P_{0 n}(t)=e^{-t} U_{n}\left(\frac{1}{\tau}\right)\left[I_{n}(\tau t)-I_{n+2}(\tau t)\right]
$$

Example 4.5 (linear rates). Let $a_{2 n}=a_{2 n+1}=n+1, n=0,1,2, \ldots$.

Using (4.1),

$$
A(m, 2 n)=m !\left(\begin{array}{c}
n+m \\
m
\end{array}\right)^{2}
$$

Therefore,

$$
\begin{aligned}
P_{0 n}(t) & =\sum_{m=0}^{\infty}(-1)^{m}\left(\begin{array}{c}
n+m \\
m
\end{array}\right) t^{n+m} \\
& =\frac{t^{n}}{(1+t)^{n}}, \quad n=0,1,2, \ldots
\end{aligned}
$$

This result agrees with Lenin et al. [8, Example 2].

Example 4.6. Let $a_{n}=(n+1) a, n=0,1,2, \ldots$, and $a>0$, then

$$
A(m, n)=\frac{(2 m) !}{2^{m} m !}\left(\begin{array}{c}
n+2 m \\
2 m
\end{array}\right) a^{m}
$$

Thus,

$$
P_{0 n}(t)=\sum_{m=0}^{\infty}(-1)^{m} \frac{(2 n+2 m) !}{m ! n !} \frac{(a t / 2)^{n+m}}{(n+m) !}, \quad n=0,1,2, \ldots .
$$

Example 4.7 (Rogers-Ramanujan CF). Consider the rates

$$
a_{n}=q^{n+1}, \quad n=0,1,2, \ldots, 0<q<1 .
$$

A BDP related to the J-fraction expansion of this has been studied by Parthasarathy et al. [13]. This CF leads to another BDP when looked as an S-fraction:

$$
f_{0}(s)=\frac{1 / s \mid}{|c|}+\frac{q / s \mid}{\mid 1}+\frac{q^{2} / s \mid}{\mid 1}+\frac{q^{3} / s}{\mid 1}+\cdots .
$$

This is the celebrated Rogers-Ramanujan CF (Berndt [2]) related to the $q$-series. Stanley 
[16, pages 235-236] has given an interesting combinatorial interpretation of this CF in terms of the area under lattice paths.

The $q$-binomial coefficient is defined as

$$
\left[\begin{array}{c}
n \\
j
\end{array}\right]= \begin{cases}\frac{(q ; q)_{n}}{(q ; q)_{j}(q ; q)_{n-j}} & \text { if } 0 \leq j \leq n \\
0 & \text { if } j<0 \text { or } j>n\end{cases}
$$

Here $(a ; q)_{n}=\prod_{i=0}^{n-1}\left(1-a q^{i}\right)$.

It is well known that

$$
\sum_{n=0}^{\infty}\left[\begin{array}{c}
m+n-1 \\
n
\end{array}\right] z^{n}=\frac{1}{(1-z)(1-z q)\left(1-z q^{2}\right) \cdots\left(1-z q^{m-1}\right)}
$$

(Andrews [1]).

Define

$$
Q_{m}(z)=\sum_{n=0}^{\infty} A(m, n) z^{n}, \quad A(0, n)=1
$$

The recurrence relation (4.1) gives

$$
Q_{m}(z)=\frac{1}{z(1-z)} Q_{m-1}(q z)-\frac{1}{z(1-z)} A(m-1,0) .
$$

From this we obtain

$$
\begin{gathered}
Q_{0}(z)=(1-z)^{-1}, \\
Q_{m}(z)=\frac{1}{z^{m} q^{\left(\begin{array}{c}
m \\
2
\end{array}\right)} \prod_{i=0}^{m}\left(1-z q^{i}\right)}-\sum_{k=0}^{m-1} \frac{A(k, 0)}{z^{m-k} q^{\left(\begin{array}{c}
m-k \\
2
\end{array}\right)} \prod_{i=0}^{m-k-1}\left(1-z q^{i}\right)} .
\end{gathered}
$$

Using (4.26),

$$
Q_{m}(z)=\frac{1}{q^{(m}\left(\begin{array}{l}
m \\
2
\end{array}\right)} \sum_{r=0}^{\infty}\left[\begin{array}{c}
m+r \\
r
\end{array}\right] z^{r-m}-\sum_{k=0}^{m-1} \frac{A(k, 0)}{q^{\left(\begin{array}{c}
m-k \\
2
\end{array}\right)}} \sum_{r=0}^{\infty}\left[\begin{array}{c}
m+r-k-1 \\
r
\end{array}\right] z^{r+k-m} .
$$

Comparing coefficients on each side, for $m \geq 1$ and $n=0,1,2, \ldots$,

$$
A(m, n)=\frac{1}{q^{\left(\begin{array}{c}
m \\
2
\end{array}\right)}}\left[\begin{array}{c}
2 m+n \\
m
\end{array}\right]-\sum_{k=0}^{m-1} \frac{1}{q^{\left(\begin{array}{c}
m-k \\
2
\end{array}\right)}}\left[\begin{array}{c}
2 m+n-2 k-1 \\
m-k-1
\end{array}\right] A(k, 0) .
$$

For $n=0$,

$$
A(m, 0)=\frac{1}{q^{(m}\left(\begin{array}{l}
m \\
2
\end{array}\right)}\left[\begin{array}{c}
2 m \\
m
\end{array}\right]-\sum_{k=0}^{m-1} \frac{1}{q^{\left(\begin{array}{c}
m-k \\
2
\end{array}\right)}}\left[\begin{array}{c}
2 m-2 k-1 \\
m-k-1
\end{array}\right] A(k, 0) .
$$


14 Transient solution of state-dependent BDPs

The above recursion leads to $A(1,0)=q$ and for $m=2,3,4, \ldots$,

$$
A(m, 0)=\left|\begin{array}{ccccc}
M_{1}(1-q) & 1 & & & \\
M_{2}\left(1-q^{2}\right) & M_{1} & 1 & & \\
M_{3}\left(1-q^{3}\right) & M_{2} & M_{1} & 1 & \\
\vdots & \vdots & \ddots & \ddots & \\
M_{m}\left(1-q^{m}\right) & M_{m-1} & M_{m-2} & \cdots & M_{1}
\end{array}\right| \text {, }
$$

where $M_{m}=q^{m^{2}} / \prod_{i=1}^{m}\left(1-q^{i}\right), m=1,2,3, \ldots$

We then obtain for $n=0,1,2, \ldots$,

$$
P_{0 n}(t)=q^{n^{2}} \sum_{m=0}^{\infty}\left\{\frac{1}{q^{\left(\begin{array}{c}
m \\
2
\end{array}\right)}}\left[\begin{array}{c}
2 m+n \\
m
\end{array}\right]-\sum_{k=0}^{m-1} \frac{1}{q^{\left(\begin{array}{c}
m-k \\
2
\end{array}\right)}}\left[\begin{array}{c}
2 m+n-2 k-1 \\
m-k-1
\end{array}\right] A(k, 0)\right\} \frac{t^{m+n}}{(m+n) !} .
$$

In the following section, we present the transient solution of a finite state-dependent BDP with examples.

\section{Finite BDPs}

For a finite state-dependent BDP with birth and death rates $a_{2 n}(0 \leq n \leq N-1)$ and $a_{2 n-1}$ $(1 \leq n \leq N)$, respectively, the transient probabilities

$$
P_{0 n}(t)=L_{n-1} \sum_{m=0}^{\infty}(-1)^{m} A(m, 2 n) \frac{t^{m+n}}{(m+n) !}, \quad n=0,1,2, \ldots, N,
$$

where

$$
A(m, n)=\sum_{i_{1}=0}^{n} a_{i_{1}} \sum_{i_{2}=0}^{i_{1}+1} a_{i_{2}} \sum_{i_{3}=0}^{i_{2}+1} a_{i_{3}} \cdots \sum_{i_{m}=0}^{i_{m-1}+1} a_{i_{m}}
$$

with $A(0, n)=1$ for every $n$, and $a_{n}=0$ if $n \geq 2 N$.

If $N=1$,

$$
f_{0}(s)=\sum_{m=0}^{\infty}(-1)^{m} \frac{A(m, 0)}{s^{n+1}}, \quad f_{1}(s)=a_{0} \sum_{m=0}^{\infty}(-1)^{m} \frac{A(m, 1)}{s^{n+2}},
$$

where

$$
\begin{gathered}
A(m, 0)=a_{0}\left(a_{0}+a_{1}\right)^{m-1}, \quad m=1,2,3, \ldots, \quad A(0,0)=1, \\
A(m, 1)=\left(a_{0}+a_{1}\right)^{m}, \quad m=0,1,2, \ldots
\end{gathered}
$$

Or,

$$
\begin{aligned}
& P_{00}(t)=\frac{a_{1}}{a_{0}+a_{1}}+\frac{a_{0}}{a_{0}+a_{1}} \exp \left[-\left(a_{0}+a_{1}\right) t\right], \\
& P_{01}(t)=\frac{a_{0}}{a_{0}+a_{1}}-\frac{a_{0}}{a_{0}+a_{1}} \exp \left[-\left(a_{0}+a_{1}\right) t\right] .
\end{aligned}
$$


If $N=2$,

$$
A(m, 0)=\frac{a_{0}}{r_{1}-r_{2}}\left[\left(r_{1}^{m}-r_{2}^{m}\right)-\left(a_{2}+a_{3}\right)\left(r_{1}^{m-1}-r_{2}^{m-1}\right)\right], \quad m=1,2,3, \ldots,
$$

where

$$
r_{1}\left(\text { or } r_{2}\right)=\frac{1}{2}\left[a_{0}+a_{1}+a_{2}+a_{3} \pm \sqrt{\left(a_{0}+a_{1}+a_{2}+a_{3}\right)^{2}-4\left(a_{0}\left(a_{2}+a_{3}\right)+a_{1} a_{3}\right)}\right]
$$

with $A(0,0)=1$, and for $m=0,1,2, \ldots$,

$$
\begin{gathered}
A(m, 2)=\frac{1}{r_{1}-r_{2}}\left[\left(r_{1}^{m+1}-r_{2}^{m+1}\right)-a_{3}\left(r_{1}^{m}-r_{2}^{m}\right)\right], \\
A(m, 3)=\frac{1}{r_{1}-r_{2}}\left[r_{1}^{m+1}-r_{2}^{m+1}\right] .
\end{gathered}
$$

We then obtain

$$
\begin{gathered}
P_{00}(t)=\left[1-\frac{a_{0}\left(a_{2}+a_{3}\right)}{r_{1} r_{2}}\right]+\frac{a_{0}}{r_{1}-r_{2}}\left[1-\frac{a_{2}+a_{3}}{r_{1}}\right] e^{-r_{1} t}+\frac{a_{0}}{r_{1}-r_{2}}\left[\frac{a_{2}+a_{3}}{r_{2}}-1\right] e^{-r_{2} t}, \\
P_{01}(t)=\frac{a_{0} a_{3}}{r_{1} r_{2}}+\frac{a_{0}}{r_{1}-r_{2}}\left[\left(\frac{a_{3}}{r_{1}}-1\right) e^{-r_{1} t}+\left(1-\frac{a_{3}}{r_{2}}\right) e^{-r_{2} t}\right], \\
P_{02}(t)=\frac{a_{0} a_{2}}{r_{1} r_{2}}+\frac{a_{0} a_{2} e^{-r_{1} t}}{r_{1}\left(r_{1}-r_{2}\right)}-\frac{a_{0} a_{2} e^{-r_{2} t}}{r_{2}\left(r_{1}-r_{2}\right)} .
\end{gathered}
$$

\section{Acknowledgment}

The first author thanks the Alexander von Humboldt Foundation for financial assistance during the preparation of this paper.

\section{References}

[1] G. E. Andrews, The Theory of Partitions, Encyclopedia of Mathematics and Its Applications, vol. 2, Addison-Wesley, Massachusetts, 1976.

[2] B. C. Berndt, Ramanujan's Notebooks. Part II, Springer, New York, 1989.

[3] P. Flajolet, Combinatorial aspects of continued fractions, Discrete Mathematics 32 (1980), no. 2, $125-161$.

[4] J. Gill, A note on extending Euler's connection between continued fractions and power series, Journal of Computational and Applied Mathematics 106 (1999), no. 2, 299-305.

[5] I. P. Goulden and D. M. Jackson, Combinatorial Enumeration, A Wiley-Interscience Publication. Wiley-Interscience Series in Discrete Mathematics, John Wiley \& Sons, New York, 1983.

[6] F. Guillemin and D. Pinchon, Excursions of birth and death processes, orthogonal polynomials, and continued fractions, Journal of Applied Probability 36 (1999), no. 3, 752-770.

[7] W. B. Jones and W. J. Thron, Continued Fractions. Analytic Theory and Applications, Encyclopedia of Mathematics and Its Applications, vol. 11, Addison-Wesley, Massachusetts, 1980.

[8] R. B. Lenin, P. R. Parthasarathy, W. R. W. Scheinhardt, and E. A. van Doorn, Families of birthdeath processes with similar time-dependent behaviour, Journal of Applied Probability 37 (2000), no. 3, 835-849. 
[9] J. A. Murphy and M. R. O'Donohoe, Some properties of continued fractions with applications in Markov processes, Journal of the Institute of Mathematics and its Applications 16 (1975), no. 1, $57-71$.

[10] P. R. Parthasarathy, A transient solution to an $M / M / 1$ queue: a simple approach, Advances in Applied Probability 19 (1987), no. 4, 997-998.

[11] Some unusual birth-and-death processes, The Mathematical Scientist 28 (2003), no. 2, 79-90.

[12] P. R. Parthasarathy and R. B. Lenin, Birth and Death Process (BDP) Models with ApplicationsQueueing, Communication Systems, Chemical Models, Biological Models: The State-of-the-Art with a Time-Dependent Perspective, American Science Press, New York, 2004.

[13] P. R. Parthasarathy, R. B. Lenin, W. Schoutens, and W. Van Assche, A birth and death process related to the Rogers-Ramanujan continued fraction, Journal of Mathematical Analysis and Applications 224 (1998), no. 2, 297-315.

[14] P. R. Parthasarathy and R. Sudhesh, A formula for the coefficients of orthogonal polynomials from the three-term recurrence relations, to appear in Applied Mathematics Letters.

[15] L. J. Rogers, On the representation of certain asymptotic series as convergent continued fractions, Proceedings of the London Mathematical Society. Second Series 4 (1907), no. 2, 72-89.

[16] R. P. Stanley, Enumerative Combinatorics. Vol. 2, Cambridge Studies in Advanced Mathematics, vol. 62, Cambridge University Press, Cambridge, 1999.

[17] R. Vein and P. Dale, Determinants and Their Applications in Mathematical Physics, Applied Mathematical Sciences, vol. 134, Springer, New York, 1999.

[18] H. S. Wall, Analytic Theory of Continued Fractions, D. Van Nostrand, New York, 1948.

[19] A. J. Zajta and W. Pandikow, Conversion of continued fractions into power series, Mathematics of Computation 29 (1975), no. 130, 566-572.

P. R. Parthasarathy: Department of Mathematics, Indian Institute of Technology Madras,

Chennai 600 036, India

E-mail address: prp@iitm.ac.in

R. Sudhesh: Department of Mathematics, Indian Institute of Technology Madras,

Chennai 600 036, India

E-mail address: rsudhesh@iitm.ac.in 


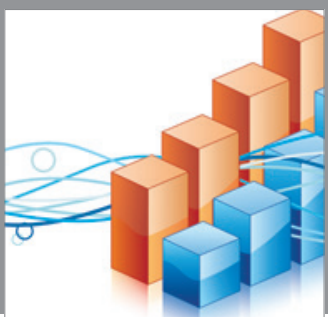

Advances in

Operations Research

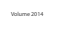

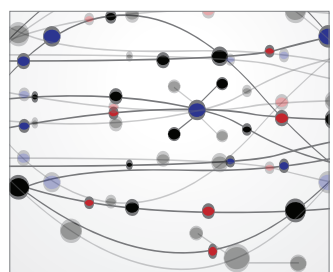

\section{The Scientific} World Journal
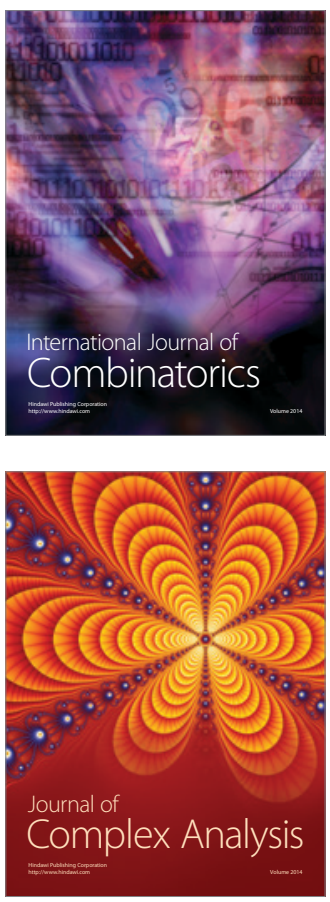

International Journal of

Mathematics and

Mathematical

Sciences
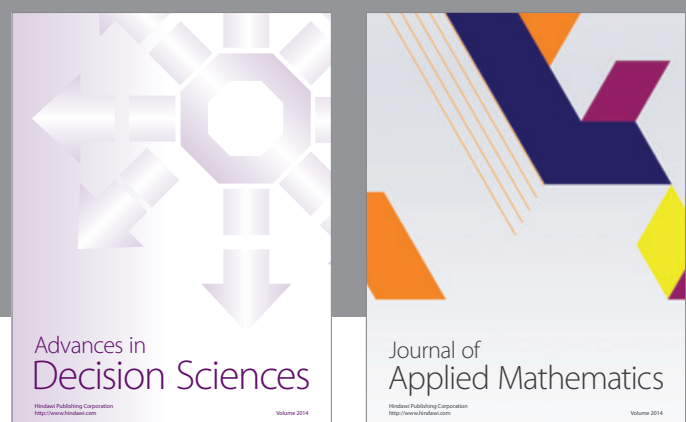

Journal of

Applied Mathematics
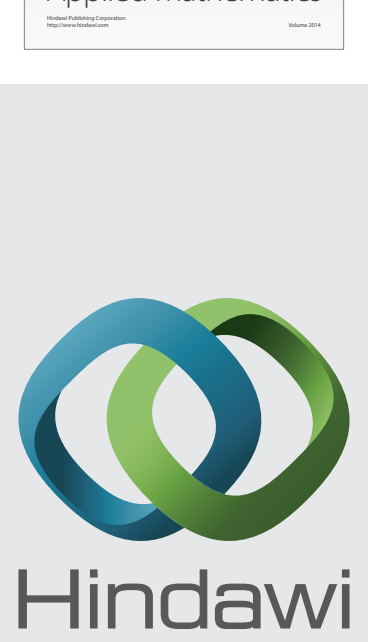

Submit your manuscripts at http://www.hindawi.com
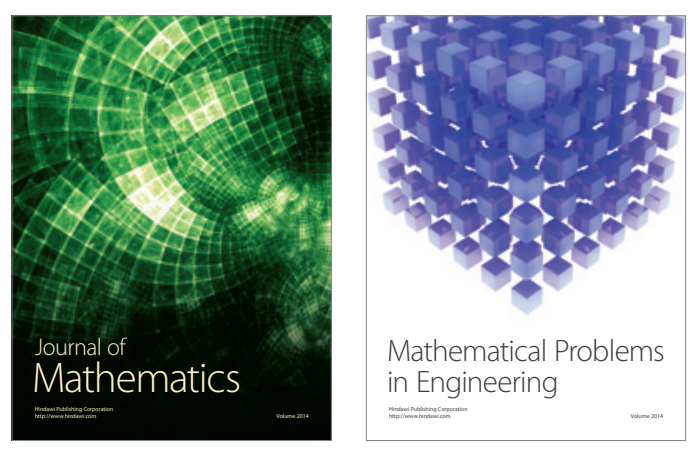

Mathematical Problems in Engineering
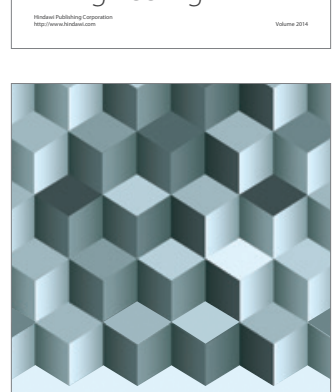

Journal of

Function Spaces
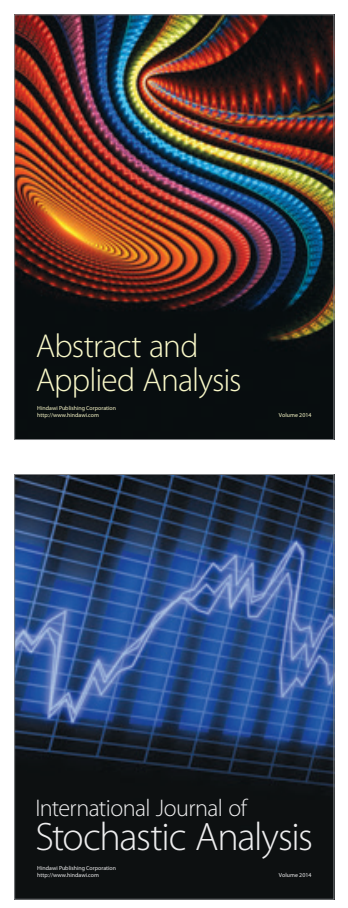

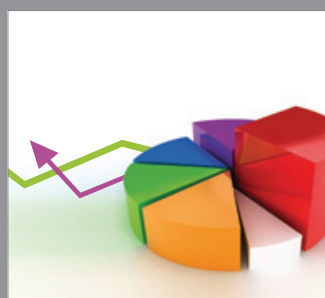

ournal of

Probability and Statistics

Promensencen
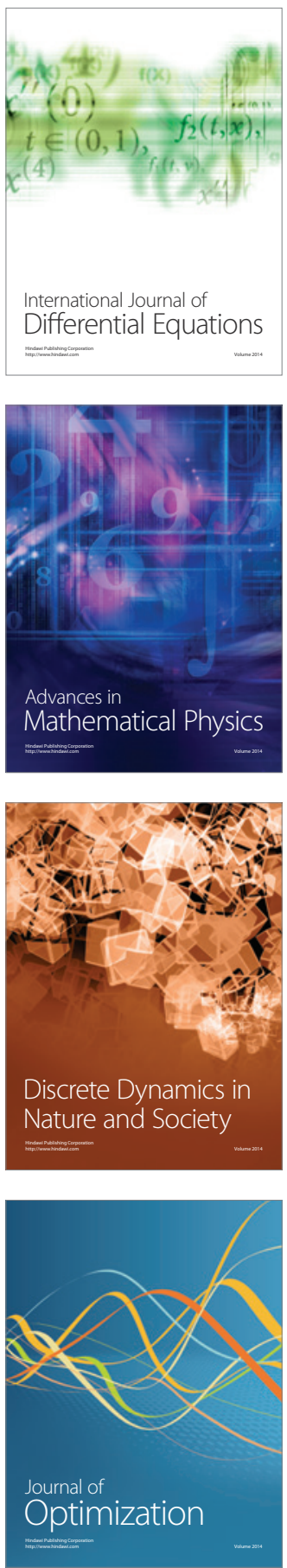